The Journal of Animal \& Plant Sciences, 31(2): 2021, Page: 358-368

ISSN (print): 1018-7081; ISSN (online): 2309-8694

\title{
PROGENY GROWTH PERFORMANCE AS INFLUENCED BY DIFFERENT PARENTAL BODY WEIGHTS IN FOUR CLOSE-BRED FLOCKS OF JAPANESE QUAILS (COTURNIX COTURNIX JAPONICA)
}

\author{
A. S. Jatoi ${ }^{* 1}$, E. Bughio ${ }^{1}$, A. W. Sahota ${ }^{2}$, M. Akram², M. H. Jaspal ${ }^{3}$, H. M. Ishaq ${ }^{4}$, J. Hussain ${ }^{2}$ and S. Mehmood ${ }^{2}$ \\ ${ }^{1}$ Department of Poultry Production, Faculty of Animal Production and Technology, Shaheed Benazir Bhutto University \\ of Veterinary and Animal Sciences, Sakrand, Pakistan, \\ ${ }^{2}$ Department of Poultry Production, ${ }^{3}$ Meat Science and Technology, Faculty of Animal Production and Technology, \\ University of Veterinary and Animal Sciences, Lahore, Pakistan \\ ${ }^{4}$ Department of Livestock \& Poultry Production, Bahauddin Zakariya University, Multan, Pakistan \\ ${ }^{1 *}$ Corresponding Author's E-mail: asultanjatoi@sbbuvas.edu.pk
}

\begin{abstract}
The present study was conducted to investigate the effect of different parental body weights on 3-weeks progeny growth performance in four close-bred flocks of Japanese quails (Coturnix coturnix japonica). A total of 432 adult (12 weeksold) quails, comprising 108 males and 324 females were randomly divided into 108 experimental units in 1:3 male to female ratio. These experimental units were randomly assigned to 12 treatment groups having 4 close-bred flocks (imported, local-1, local-2 and local-3) $\times 3$ female body weights (heavy 300-350g, medium 250-300g and small 200$250 \mathrm{~g}$ ) with Randomized Complete Block Design (RCBD) in factorial arrangements having 9 replicates in each treatment. The different parental body weight categories significantly $(\mathrm{p}<0.05)$ affected day-old, $1^{\text {st }}, 2^{\text {nd }}$ and $3^{\text {rd }}$ weeks progeny body weight in Japanese quails. The heavy male parents had apparently more pronounced effect on day-old and $1^{\text {st }}$ week progeny body weight. The cumulative progeny body weight gain in quails of different close-flocks differed significantly $(p<0.05)$ in all the parental groups. The interaction between parental body size and close-bred flocks was significant $(\mathrm{p}<0.05)$. Effect of different parental body size on $1^{\text {st }}, 2^{\text {nd }}$, and $3^{\text {rd }}$ week cumulative progeny body weight gain, feed intake, FCR and mortality rates were also significant $(\mathrm{p}<0.05)$. The interaction between parental body size and closebred flocks was significant for these parameters. In conclusion, progeny secured from heavy male parent had higher hatch weight, body weight, weight gain and feed intake than those hatched from medium and small male parents, showing more pronounced effect of male parent on progeny growth performance and on almost all the other parameters.
\end{abstract}

Key words: Japanese quail, body weight, weight gain, close-bred, feed intake, FCR, mortality percent. https://doi.org/10.36899/JAPS.2021.2.0223

Published online October 03,2020

\section{INTRODUCTION}

Japanese quail considered as the best avian model and pilot bird in the field of research due to their faster growth rate, early sexual maturity, shorter generation interval, high rate of egg production, less feed consumption, easy and cheap maintenance, relatively easy to handle and resistance to many tropical diseases (Minvielle, 2004; Dhaliwal et al., 2004; Narinc et al., 2010; Daikwao et al., 2013; Onyewuchi et al., 2013; Saidu et al., 2014). Since centuries; Japanese quail is considered as one of the most important source for meat and egg production in many European and Asian countries (Maiorano et al., 2012; Ayasan, 2013; Tunsaringkarn et al., 2013). Quail farming is also one of the most suitable and low capital investment, rapid return and easily adaptable enterprise all over the world including Pakistan. However, due to lack of knowledge, shortage of breeding stocks and good quality of day old chicks, Japanese quail is not emerging as a profitable enterprise. The quail meat is not as popular as chicken meat in the country. It is usually consumed as a special dish and at higher rates than chicken meat. The public and private sectors had taken measures for the development of quail farming as an industry, but such measures were not adequate and fall short of expectations for producing high yield of quail meat at a reasonable low cost. The technical reason for the slow development of the quail farming is the lack of application of modern quail production technology, particularly at small scale farmers (Akram et al., 2008). Growth rate at different ages are useful selection criteria and important tool in most of the breeding programs in animal production; i.e. increase live body weight, weight gain, feed intake and mortality during early period of life (Hassan, 2011; Udeh and Omeje, 2011; Hussen et al., 2016; Fadhil and Hassan, 2018).

Despite having enormous potential, very little research has been conducted on the acclimatization of Japanese quails to Pakistan's environment. Keeping above in view these issues, four different close-bred local and imported Japanese quail flocks have been maintained 
at Avian Research and Training Centre, Department of Poultry Production, University of Veterinary and Animal Sciences Lahore, Pakistan, with objectives of making attempts to improve productive and growth potentials of quails. The present study was planned to investigate the growth performance of progenies obtained from these close-bred flocks of Japanese quails of different parental body sizes up to the age of 3-weeks.

\section{MATERIALS AND METHODS}

The present study was conducted at Avian Research and Training Centre, University of Veterinary and Animal Sciences, Lahore, Pakistan. A total of 432 adult (12 weeks-old) quails, comprising 108 males and 324 females were used. The birds were randomly selected from the available stock and then divided into 108 experimental units (replicates comprising one male and three females of each.) These experimental units were randomly assigned to 12 treatment groups having 4 closebred flocks by designating their name as Imported (Major), Local-1 (Kaleem) Local-2 (Saadat) and Local-3 (Zahid) $\times 3$ female body weights with randomized complete block design in factorial arrangements having 9 replicates in each treatment. Further procedure was assumed after Jatoi et al. (2015a). On completion of hatching, day-old quail chicks from each replicate were weighed individually by using sophisticated digital balance. The chicks in each replicate were placed in French made brooding batteries under standard management conditions. The quail chicks were fed a balanced quail broiler starter ration ad-libitum (broiler starter crumbs feed grinded into mash form). The ration was formulated and prepared at Hi-Tech Feeds PVT (Ltd), Lahore, according to NRC standards (1994) (Table 1). The birds had a free access to clean and fresh drinking water through drinking nipple lines. The brooding temperature in battery cages for first week was maintained between $31^{\circ} \mathrm{C}$ and $35^{\circ} \mathrm{C}$ and then weekly reduced by $3^{\circ} \mathrm{C}$ up to the age of 3 weeks (North and Bell, 1991). The experimental birds were tagged for their proper identification. Initial body weight at hatching and thereafter weekly body weight of quail chicks up to 3 weeks were recorded. The progeny growth parameters (day-old quail chicks weight (g), body weight (g), weight gain (g), feed intake (g/bird), FCR (feed/g gain) and mortality rate $(\%)$ were recorded up to the age of 3 weeks. Feed conversion ratio (FCR) was worked out for individual chicks on the basis of body weight gain and feed intake,

Statistical Analysis: The data collected were analyzed using ANOVA techniques with RCBD, with more than one observation for further interpretation using general linear model procedures (SAS 9.1, 2002-03), assuming the following mathematical model: (The mathematical model was assumed after Jatoi et al. (2015b)). The comparison of means was made using Duncan's Multiple Range (DMR) test (Duncan 1955).

Mathematical model:

$\mathrm{Yijkl}=\mu+\mathrm{Fi}+\mathrm{Sj}+\mathrm{Wk}+\mathrm{Sj} \times \mathrm{Wk}+$ eijkl, where:

Yijkl = the 1 th observation of the $\mathrm{k} t h$ category of females of the $\mathrm{j}$ th category of males of the ith flock;

$\mu=$ population mean;

$\mathrm{Fi}=$ effect of the $\mathrm{i}$ th flock $(\mathrm{i}=4)$, treated as blocks;

$\mathrm{Sj}=$ effect of the $\mathrm{j}$ th category of male $(\mathrm{k}=3)$;

$\mathrm{Wk}=$ effect of the kth category of female $(\mathrm{j}=3)$;

eijk $=$ random error associated with the ith flock and $\mathrm{j}$ th body weight category.

\section{RESULTS AND DISCUSSION}

Body weight (g): In the present study, different parental body weight categories significantly $(p<0.05)$ affected day-old progeny body weight and also $1^{\text {st }}, 2^{\text {nd }}$ and $3^{\text {rd }}$ week body weight of Japanese quail. The heavy male parents had apparently more pronounced effect on dayold and $1 \mathrm{~s}$ t week progeny body weights. The interaction between parental body size and close-bred flocks was significant $(\mathrm{p}<0.05)$ for $1 \mathrm{st}, 2$ nd and 3 rd weeks progeny body weight except for day-old body weight (Table 2). The findings of this study indicating effect of parent body weight on progeny body weight could be due to higher day-old chick weight from heavy parents which subsequently lead to higher final body weight at $3 \mathrm{rd}$ week in the progeny. The similar findings indicating significant $(p<0.05)$ effect of hatch weight on 2 nd week body weight in quails have been reported (Saatci et al., 2003; Saatci et al., 2006; Shokoohmand et al., 2007; Kumari et al., 2009; Alkan et al., 2010; Lotfi et al., 2012). The earlier findings also indicated that maternal effect on chick weight was possibly mediated via egg composition of both the genetic and the environmental origin (Hartmann et al., 2003; Dogan et al., 2010; Hussen et al., 2016). Furthermore; similar strain variation in average weekly body weight in broiler at 4-week of age has also been indicated by Yakubu et al. (2010). Hussain et al., (2013) also reported significant improvement in body weight $(\mathrm{g})$ in selected groups of Japanese quails at the age of 21 days. Dudusola (2013) reported that parental age was found to have a significant effect on the weight of dayold chick was found to be significant $(\mathrm{P}<0.05)$. It was also observed that with increasing egg weight, chick weight increased. Wang et al., (2019) also reported that male chicken had better growth performance than female chickens. Furthermore Krishna and Sahitya Rani (2017) reported that body weight of the progeny obtained from baseline and selected female quail population was found to be higher than the parental population body weights at the end of the growth period. Significant effect of sex and line $(P<0.001)$ was also observed by Khaldari et al., 
(2010) in Japanese quail selected for 4-week body weight.

Weight gain (g): In the present study, effect of different parental body size on 1st, 2nd and 3rd week and cumulative progeny body weight gain was found to be significant $(\mathrm{p}<0.05)$. The interaction between parental body size and close-bred flocks was also significant (Table 3). The results of this study shows variation in body weight gain among different close-bred flocks are in agreement with the earlier findings of Yakubu et al., (2010) who reported strain variation $(p<0.05)$ in body weight gain in broilers at the age of 4-week. Furthermore; similar strain variation in body weight gain in Aseel chicken at different ages has also been indicated by Iqbal (2011). Hussain et al., (2013) also reported significant improvement in body weight gain $(\mathrm{g})$ in selected groups of Japanese quails at the age of 21 days. Similarly, Krishna and Sahitya Rani (2017) also reported that the body weight gain was found to be higher in the progeny than the parental population at six weeks of age.

Feed intake (g) and FCR: In the present study, $1^{\text {st }}, 2^{\text {nd }}$ and $3^{\text {rd }}$ week and cumulative feed intake and feed conversion ratio of the progeny were significantly $(p<0.05)$ influenced by parental body size of Japanese quails. The interaction between parental body size and close-bred flocks was significant $(\mathrm{p}<0.05)$ (Table 4 and 5 ). These results indicated variation in FCR in quail progenies from different close-bred flocks. Similar to present findings; Sahota et al., (2003) reported significant $(p<0.01)$ differences in feed conversion efficiency in progenies of Desi chickens in comparison to their parents. Similar findings of Yakubu et al., (2010) who reported that strain variation $(\mathrm{p}<0.05)$ in average weekly feed intake in broilers at the age of 4 -week. Hussain et al., (2013) also reported significant improvement in feed intake (g) and FCR in selected groups of Japanese quails at the age of 21 days. The FCR in four varieties of Aseel was significantly $(\mathrm{p}<0.05)$ different at $1 \mathrm{st}, 5 \mathrm{th}, 6 \mathrm{th}, 7 \mathrm{th}$, 8th, 9th, 10th, 11th, 12th, 13th, 14th and 15th weeks of age (Iqbal 2011). The findings of the present study showing effect of parental body weight on progeny feed intake in quails are fully supported by those of Renden and McDaniel (1984) and Farooq (1989) who reported that daily feed intake was significantly $(\mathrm{p}<0.05)$ different between heavy and light hens and were directly related to their body weight. The maintenance requirement of feed has been reported to be increased with increase in body weight of birds which reduced availability of energy required for their growth (Rondelli et al., 2003; Varkoohi et al., 2010; Okenyi et al., 2013). Krishna and Sahitya Rani (2017) also reported that feed conversion ratio of the progeny was found to be better feed conversion ratio compared with baseline and selected population during the growth period.
Mortality rate (\%): In the present study, a significant $(p<0.05)$ effect of different parental body weights on progeny mortality rate (\%) during $1 \mathrm{st}, 2$ nd and $3 \mathrm{rd}$ week and cumulative progeny mortality rate was recorded. The mortality rate in quail progenies secured from small size parents was higher than those hatched from heavy and medium parents. The interaction between parental groups and close-bred flocks was significant $(\mathrm{p}<0.05)$ (Table 6). This high mortality rate could be attributed to small egg and chick size from small parents. These results agree with those of Among et al., (1984) and Farooq (1989) who reported higher mortality rate in chicks hatched from smaller eggs than of larger eggs. Wilson (1991, 1991a) indicated that weight of the newly hatched chick was correlated with post-hatch growth and chick mortality. Yassin et al., (2009) reported significant differences in first week mortality in broilers hatched from different broiler breeders. Livability in broilers may depend on day-old chick quality and farm management practices (Goodhope 1991; Wilson 1991a; El-Fiky et al., 2000; Joseph and Moran 2005; Tona et al., 2005; Decuypere and Bruggeman 2007; Umar et al., 2013). Hussain et al., (2013) also reported low mortality rate in selected groups of Japanese quails at the age of 21 days. Munisi et al., (2015) reported that there were no differences in livability amongst genetic stocks.

Table-1. Feed composition.

\begin{tabular}{cc}
\hline Ingredients & \% \\
\hline Maize & 50.0 \\
Rice Polish & 6.00 \\
Canola Meal & 1.98 \\
Soybean Meal & 30.54 \\
Corn Gluten 60\% & 6.00 \\
Lime stone & 1.11 \\
D-L Methionine & 0.11 \\
L- Lysine & 0.22 \\
Threonine & 0.15 \\
DCP & 1.28 \\
Vitamin Supplement & 1.30 \\
Rock Salt & 0.30 \\
Composition of nutrients \\
\hline NUTRIENTS & VALUES \\
\hline ME Kcal/kg & 2900 \\
CP \% & 24.00 \\
Ca \% & 0.80 \\
Available P \% & 0.30 \\
Phytate P \% & 0.34 \\
Total P \% & 0.65 \\
Crude Fiber \% & 4.38 \\
Linoleic acid \% & 1.42 \\
Methionine \% & 0.50 \\
Lysine \% & 1.30 \\
\hline
\end{tabular}


Table-2. Day-old, $1^{\text {st }}, 2^{\text {nd }}$ and $3^{\text {rd }}$ week progeny body weight (g) influenced by 3 parental body weight categories from 4 close-bred flocks of Japanese quails.

\begin{tabular}{|c|c|c|c|c|c|c|c|c|c|}
\hline \multirow[t]{2}{*}{$\begin{array}{l}1 \\
0 \\
0 \\
+\end{array}$} & \multicolumn{3}{|c|}{ Heavy } & \multicolumn{3}{|c|}{ Medium } & \multicolumn{3}{|c|}{ Small } \\
\hline & Heavy & Medium & Small & Heavy & Medium & Small & Heavy & Medium & Small \\
\hline Imported & $\begin{array}{l}8.00 \\
\pm 0.11^{\mathrm{abA}}\end{array}$ & $\begin{array}{l}7.71 \\
\pm 0.18^{\text {abcdA }}\end{array}$ & $\begin{array}{l}7.91 \\
\pm 0.15^{\mathrm{abcA}}\end{array}$ & $\begin{array}{l}7.60 \\
\pm 0.14^{\mathrm{abcdA}}\end{array}$ & $\begin{array}{l}\left.\mathbf{E}^{*} ; \mathbf{D O}^{* *} \mathbf{g}\right) \\
7.33 \\
\pm 0.12^{\mathrm{abcdA}}\end{array}$ & $\begin{array}{l}7.49 \\
\pm 0.16^{\text {abcdA }}\end{array}$ & $\begin{array}{l}7.55 \\
\pm 0.18^{\mathrm{abcdA}}\end{array}$ & $\begin{array}{l}7.46 \\
\pm 0.10^{\mathrm{abcdA}}\end{array}$ & $\begin{array}{l}7.30 \\
\pm 0.25^{\mathrm{bcdA}}\end{array}$ \\
\hline Local-1 & $\begin{array}{l}7.66 \\
\pm 0.34^{\text {abcdA }}\end{array}$ & $\begin{array}{l}7.43 \\
\pm 0.23^{\text {abcdA }}\end{array}$ & $\begin{array}{l}7.64 \\
\pm 0.27^{\text {abcdA }}\end{array}$ & $\begin{array}{l}7.74 \\
\pm 0.12^{\text {abcdA }}\end{array}$ & $\begin{array}{l}7.35 \\
\pm 0.49^{\text {abcdA }}\end{array}$ & $\begin{array}{l}7.58 \\
\pm 0.04^{\mathrm{abcdA}}\end{array}$ & $\begin{array}{l}7.51 \\
\pm 0.16^{\text {abcdA }}\end{array}$ & $\begin{array}{l}7.50 \\
\pm 0.17^{\text {abcdA }}\end{array}$ & $\begin{array}{l}7.61 \\
\pm 0.10^{\text {abcdA }}\end{array}$ \\
\hline Local-2 & $\begin{array}{l}7.98 \\
\pm 0.19^{\mathrm{abcA}}\end{array}$ & $\begin{array}{l}7.41 \\
\pm 0.64^{\mathrm{abcdA}}\end{array}$ & $\begin{array}{l}7.68 \\
\pm 0.25^{\text {abcdA }}\end{array}$ & $\begin{array}{l}7.85 \\
\pm 0.06^{\mathrm{abcA}}\end{array}$ & $\begin{array}{l}7.50 \\
\pm 0.18^{\mathrm{abcdA}}\end{array}$ & $\begin{array}{l}7.64 \\
\pm 0.16^{\mathrm{abcdA}}\end{array}$ & $\begin{array}{l}7.40 \\
\pm 0.10^{\mathrm{abcdA}}\end{array}$ & $\begin{array}{l}7.19 \\
\pm 0.07^{\mathrm{bcdA}}\end{array}$ & $\begin{array}{l}7.25 \\
\pm 0.34^{\mathrm{bcdA}}\end{array}$ \\
\hline Local-3 & $\begin{array}{l}7.70 \\
\pm 0.21^{\text {abcdA }}\end{array}$ & $\begin{array}{l}7.15 \\
\pm 0.44^{\text {cdA }}\end{array}$ & $\begin{array}{l}8.14 \\
\pm 0.23^{\mathrm{aA}}\end{array}$ & $\begin{array}{l}7.83 \\
\pm 0.26^{\mathrm{abcA}}\end{array}$ & $\begin{array}{l}7.74 \\
\pm 0.23^{\mathrm{abcdA}}\end{array}$ & $\begin{array}{l}7.28 \\
\pm 0.13^{\mathrm{bcdA}}\end{array}$ & $\begin{array}{l}7.69 \\
\pm 0.03^{\text {abcdA }}\end{array}$ & $\begin{array}{l}6.98 \\
\pm 0.20^{\mathrm{dA}}\end{array}$ & $\begin{array}{l}7.24 \\
\pm 0.09^{\mathrm{bcdA}}\end{array}$ \\
\hline Imported & $\begin{array}{l}27.77 \\
\pm 0.99^{\mathrm{abcdA}}\end{array}$ & $\begin{array}{l}27.96 \\
\pm 1.19^{\mathrm{abcA}}\end{array}$ & $\begin{array}{l}27.64 \\
\pm 0.52^{\text {abcdeA }}\end{array}$ & $\begin{array}{l}25.92 \\
\pm 1.58^{\text {abcdeA }}\end{array}$ & $\begin{array}{l}\left.\mathbf{F}^{*} \mathbf{1}^{\text {st }} \mathbf{w k}^{\dagger} \mathbf{g} \mathbf{g}\right)- \\
28.49 \\
\pm 1.66^{\mathrm{aA}}\end{array}$ & $\begin{array}{l}25.38 \\
\pm 0.85^{\text {abcdeA }}\end{array}$ & $\begin{array}{l}27.78 \\
\pm 1.26^{\text {abcdA }}\end{array}$ & $\begin{array}{l}28.04 \\
\pm 2.98^{\mathrm{abA}}\end{array}$ & $\begin{array}{l}26.72 \\
\pm 0.62^{\text {abcdeA }}\end{array}$ \\
\hline Local-1 & $\begin{array}{l}26.23 \\
\pm 1.55^{\text {abcdeA }}\end{array}$ & $\begin{array}{l}24.89 \\
\pm 1.12^{\text {abcdeA }}\end{array}$ & $\begin{array}{l}26.55 \\
\pm 0.50^{\text {abcdeA }}\end{array}$ & $\begin{array}{l}26.37 \\
\pm 0.23^{\text {abcdeA }}\end{array}$ & $\begin{array}{l}26.27 \\
\pm 1.17^{\mathrm{abcdeA}}\end{array}$ & $\begin{array}{l}26.90 \\
\pm 0.35^{\text {abcdeA }}\end{array}$ & $\begin{array}{l}25.50 \\
\pm 0.58^{\text {abcdeA }}\end{array}$ & $\begin{array}{l}26.57 \\
\pm 0.65^{\text {abcdeA }}\end{array}$ & $\begin{array}{l}25.09 \\
\pm 0.21^{\text {abcdeA }}\end{array}$ \\
\hline Local-2 & $\begin{array}{l}27.54 \\
\pm 0.53^{\text {abcdeA }}\end{array}$ & $\begin{array}{l}23.85 \\
\pm 1.80^{\mathrm{eB}}\end{array}$ & $\begin{array}{l}23.96 \\
\pm 1.50^{\mathrm{deB}}\end{array}$ & $\begin{array}{l}27.01 \\
\pm 0.58^{\text {abcdeA }}\end{array}$ & $\begin{array}{l}25.69 \\
\pm 0.15^{\text {abcdeA }}\end{array}$ & $\begin{array}{l}26.26 \\
\pm 0.19^{\text {abcdeA }}\end{array}$ & $\begin{array}{l}24.10 \\
\pm 0.37^{\mathrm{deB}}\end{array}$ & $\begin{array}{l}23.81 \\
\pm 0.45^{\mathrm{eB}}\end{array}$ & $\begin{array}{l}24.02 \\
\pm 1.35^{\mathrm{deA}}\end{array}$ \\
\hline Local-3 & $\begin{array}{l}25.69 \\
\pm 1.18^{\text {abcdeA }}\end{array}$ & $\begin{array}{l}24.18 \\
\pm 2.15^{\text {cdeAB }}\end{array}$ & $\begin{array}{l}27.41 \\
\pm 0.33^{\text {abcde A }}\end{array}$ & $\begin{array}{l}25.98 \\
\pm 0.63^{\text {abcdeA }}\end{array}$ & $\begin{array}{l}25.25 \\
\pm 0.59^{\text {abcdeA }}\end{array}$ & $\begin{array}{l}24.83 \\
\pm 1.18^{\text {abcdeA }}\end{array}$ & $\begin{array}{l}24.55 \\
\pm 0.60^{\text {bcdeAB }}\end{array}$ & $\begin{array}{l}24.02 \\
\pm 0.29^{\mathrm{deAB}}\end{array}$ & $\begin{array}{l}24.35 \\
\pm 0.21^{\text {bcdeA }}\end{array}$ \\
\hline Imported & $\begin{array}{l}58.80 \\
\pm 1.23^{\text {abcdeA }}\end{array}$ & $\begin{array}{l}62.10 \\
\pm 1.73^{\text {abcdeA }}\end{array}$ & $\begin{array}{l}60.04 \\
\pm 1.63^{\mathrm{abcdeA}}\end{array}$ & $\begin{array}{l}60.01 \\
\pm 4.06^{\text {abcdeA }}\end{array}$ & $\begin{array}{l}\left.{ }^{*} ; \mathbf{2}^{\mathbf{n d}} \mathbf{w k}^{\dagger} \mathbf{g}\right) \\
58.92 \\
\pm 0.68^{\text {abcdeA }}\end{array}$ & $\begin{array}{l}55.88 \\
\pm 3.09^{\mathrm{bcdeA}}\end{array}$ & $\begin{array}{l}60.69 \\
\pm 3.55^{\text {abcdeA }}\end{array}$ & $\begin{array}{l}59.21 \\
\pm 3.59^{\text {abcdeA }}\end{array}$ & $\begin{array}{l}54.02 \\
\pm 3.06^{\mathrm{cdeA}}\end{array}$ \\
\hline Local-1 & $\begin{array}{l}55.58 \\
\pm 1.64^{\text {bcdeA }}\end{array}$ & $\begin{array}{l}57.28 \\
\pm 2.13^{\text {abcdeA }}\end{array}$ & $\begin{array}{l}61.09 \\
\pm \mathrm{a} 1.52^{\mathrm{bcdA}}\end{array}$ & $\begin{array}{l}60.79 \\
\pm 2.29^{\text {abcdeA }}\end{array}$ & $\begin{array}{l}55.09 \\
\pm 3.18^{\text {bcdeA }}\end{array}$ & $\begin{array}{l}65.51 \\
\pm 0.89^{\mathrm{aA}}\end{array}$ & $\begin{array}{l}57.59 \\
\pm 0.82^{\text {abcdeA }}\end{array}$ & $\begin{array}{l}59.26 \\
\pm 1.81^{\text {abcdeA }}\end{array}$ & $\begin{array}{l}54.56 \\
\pm 0.93^{\text {bcdeB }}\end{array}$ \\
\hline Local-2 & $\begin{array}{l}62.71 \\
\pm 1.20^{\text {abcA }}\end{array}$ & $\begin{array}{l}52.92 \\
\pm 5.49^{\mathrm{deB}}\end{array}$ & $\begin{array}{l}56.05 \\
\pm 2.62^{\text {bcdeA }}\end{array}$ & $\begin{array}{l}61.39 \\
\pm 1.38^{\mathrm{abcA}}\end{array}$ & $\begin{array}{l}58.38 \\
\pm 2.20^{\text {abcdeA }}\end{array}$ & $\begin{array}{l}58.97 \\
\pm 1.55^{\text {abcdeA }}\end{array}$ & $\begin{array}{l}55.79 \\
\pm 0.76^{\text {bdeA }}\end{array}$ & $\begin{array}{l}52.62 \\
\pm 0.77^{\mathrm{eB}}\end{array}$ & $\begin{array}{l}54.05 \\
\pm 2.99^{\text {cdeB }}\end{array}$ \\
\hline Local-3 & $\begin{array}{l}60.20 \\
\pm \mathrm{a} 2.36^{\text {bdeA }}\end{array}$ & $\begin{array}{l}55.80 \\
\pm 4.56^{\text {bdeAB }}\end{array}$ & $\begin{array}{l}60.40 \\
\pm 1.02^{\text {abcdeA }}\end{array}$ & $\begin{array}{l}57.91 \\
\pm 1.06^{\text {abcdeA }}\end{array}$ & $\begin{array}{l}53.85 \\
\pm 2.85^{\mathrm{cdeB}}\end{array}$ & $\begin{array}{l}54.57 \\
\pm 1.92^{\text {bcdeB }}\end{array}$ & $\begin{array}{l}61.21 \\
\pm 1.70^{\text {abcdA }}\end{array}$ & $\begin{array}{l}54.59 \\
\pm 0.74^{\text {bcdeAB }}\end{array}$ & $\begin{array}{l}56.86 \\
\pm 0.85^{\text {bcdeB }}\end{array}$ \\
\hline Imported & $\begin{array}{l}105.53 \\
\pm 2.83^{\text {abcdA }}\end{array}$ & $\begin{array}{l}102.13 \\
\pm 2.07^{\text {bdefa }}\end{array}$ & $\begin{array}{l}104.53 \\
\pm 3.07^{\text {abcdA }}\end{array}$ & $\begin{array}{l}111.91 \\
\pm 4.26^{\mathrm{abA}}\end{array}$ & $\begin{array}{l}\left.{ }^{*} ; \mathbf{3}^{\text {rd }} \mathbf{w k}^{\dagger} \mathbf{g}\right) \\
104.69 \\
\pm 5.11^{\mathrm{abcdA}}\end{array}$ & $\begin{array}{l}99.18 \\
\pm 3.66^{\text {cdefA }}\end{array}$ & $\begin{array}{l}113.52 \\
\pm 3.96^{\mathrm{aA}}\end{array}$ & $\begin{array}{l}103.61 \\
\pm 3.60^{\text {abcdefA }}\end{array}$ & $\begin{array}{l}95.15 \\
\pm 4.26^{\text {defA }}\end{array}$ \\
\hline Local-1 & $\begin{array}{l}99.58 \\
\pm 1.23^{\text {cdefB }}\end{array}$ & $\begin{array}{l}103.61 \\
\pm 1.35^{\text {abcdefA }}\end{array}$ & $\begin{array}{l}101.79 \\
\pm 2.48^{\text {bcdefA }}\end{array}$ & $\begin{array}{l}103.85 \\
\pm 2.49^{\text {abcdefB }}\end{array}$ & $\begin{array}{l}97.09 \\
\pm 4.60^{\text {cdefB }}\end{array}$ & $\begin{array}{l}106.68 \\
\pm 4.24^{\mathrm{abcB}}\end{array}$ & $\begin{array}{l}101.72 \\
\pm 2.20^{\text {bcdefB }}\end{array}$ & $\begin{array}{l}102.42 \\
\pm 1.62^{\text {abcdefA }}\end{array}$ & $\begin{array}{l}94.48 \\
\pm 3.59^{\operatorname{def} \mathrm{A}}\end{array}$ \\
\hline Local-2 & $\begin{array}{l}105.51 \\
\pm 3.65^{\mathrm{abcdA}}\end{array}$ & $\begin{array}{l}96.65 \\
\pm 5.44^{\text {cdefA }}\end{array}$ & $\begin{array}{l}97.02 \\
\pm 3.78^{\mathrm{cdefB}}\end{array}$ & $\begin{array}{l}106.99 \\
\pm 4.79^{\mathrm{abc} A B}\end{array}$ & $\begin{array}{l}101.18 \\
\pm 2.17^{\text {bcdefAB }}\end{array}$ & $\begin{array}{l}101.90 \\
\pm 2.61^{\text {bcdefAB }}\end{array}$ & $\begin{array}{l}100.46 \\
\pm 0.44^{\text {cdefB }}\end{array}$ & $\begin{array}{l}93.05 \\
\pm 3.57^{\mathrm{efB}}\end{array}$ & $\begin{array}{l}92.68 \\
\pm 3.76 f^{\mathrm{B}}\end{array}$ \\
\hline Local-3 & $\begin{array}{l}104.53 \\
\pm 2.45^{\text {abcdA }}\end{array}$ & $\begin{array}{l}98.40 \\
\pm 4.78^{\text {cdefA }}\end{array}$ & $\begin{array}{l}103.13 \\
\pm 0.50^{\text {bcdefAB }}\end{array}$ & $\begin{array}{l}104.47 \\
\pm 3.36^{\text {abcdeA }}\end{array}$ & $\begin{array}{l}94.88 \mathrm{~d} \\
\pm 1.74^{\mathrm{efB}}\end{array}$ & $\begin{array}{l}96.34 \\
\pm 2.53^{\operatorname{cdefAB}}\end{array}$ & $\begin{array}{l}107.13 \\
\pm 2.90^{\mathrm{abc} A \mathrm{~B}}\end{array}$ & $\begin{array}{l}97.03 \\
\pm 1.43^{\text {cdef } A B}\end{array}$ & $\begin{array}{l}98.22 \\
\pm 1.88^{\text {cdefAB }}\end{array}$ \\
\hline
\end{tabular}

Different small alphabets on means in a row show significant differences at $\mathrm{p}<0.05$

Different capital alphabets on means in a column show significant differences at $\mathrm{p}<0.05$

${ }^{*} \mathrm{SE}=$ Standard error

${ }^{* *} \mathrm{DO}=$ Day-old

${ }^{*} \mathrm{wk}=$ Week 
Table-3. $1^{\text {st }}, 2^{\text {nd }}, 3$ rd and 3-week progeny weight gain (g) influenced by 3 parental body weight categories from 4 close-bred flocks of Japanese quails.

\begin{tabular}{|c|c|c|c|c|c|c|c|c|c|}
\hline \multirow{2}{*}{$\begin{array}{l}1 \\
0 \\
9 \\
\end{array}$} & \multicolumn{3}{|c|}{ Heavy } & \multicolumn{3}{|c|}{ Medium } & \multicolumn{3}{|c|}{ Small } \\
\hline & Heavy & Medium & Small & Heavy & Medium & Small & Heavy & Medium & Small \\
\hline & & & & ------. & Mean $\pm \mathrm{SE}^{*}$ & wk $^{* * *}$ g) ---- & -------- & & \\
\hline \multirow[t]{2}{*}{ Imported } & 19.76 & 20.24 & 19.73 & 18.32 & 21.16 & 17.89 & 20.22 & 20.57 & 19.41 \\
\hline & $\pm 0.90^{\mathrm{abcd} A}$ & $\pm 1.03^{\mathrm{abcA}}$ & $\pm 0.40^{\mathrm{abcdA}}$ & $\pm 1.44^{\mathrm{abcdA}}$ & $\pm 1.58^{\mathrm{aA}}$ & $\pm 0.74^{\mathrm{abcdA}}$ & $\pm 1.14^{\mathrm{abcA}}$ & $\pm 2.99^{\mathrm{abA}}$ & $\pm 0.81^{\mathrm{abcdA}}$ \\
\hline \multirow[t]{2}{*}{ Local-1 } & 18.57 & 17.36 & 18.91 & 18.63 & 18.92 & 19.32 & 17.99 & 19.07 & 17.47 \\
\hline & $\pm 1.36^{\mathrm{abcdA}}$ & $\pm 0.89^{\mathrm{bcdB}}$ & $\pm 0.77^{\mathrm{abcdA}}$ & $\pm 0.22^{\mathrm{abcdA}}$ & $\pm 0.74^{\mathrm{abcdA}}$ & $\pm 0.40^{\mathrm{abcdA}}$ & $\pm 0.48^{\mathrm{abcdA}}$ & $\pm 0.40^{\mathrm{abcdA}}$ & $\pm 0.29^{\mathrm{bcdA}}$ \\
\hline \multirow[t]{2}{*}{ Local-2 } & 19.55 & 16.44 & 16.28 & 19.16 & 18.19 & 18.62 & 16.69 & 16.61 & 16.77 \\
\hline & $\pm 0.69^{\mathrm{abcdA}}$ & $\pm 1.45^{\mathrm{dB}}$ & $\pm 1.75^{\mathrm{dB}}$ & $\pm 0.51^{\mathrm{abcdA}}$ & $\pm 0.05^{\mathrm{abcdA}}$ & $\pm 0.24^{\mathrm{abcd} A}$ & $\pm 0.33^{\mathrm{cdB}}$ & $\pm 0.39^{\mathrm{dB}}$ & $\pm 1.05^{\mathrm{cdAB}}$ \\
\hline \multirow[t]{3}{*}{ Local-3 } & 17.99 & 17.03 & 19.27 & 18.14 & 17.51 & 17.54 & 16.86 & 17.03 & 17.10 \\
\hline & $\pm 1.27^{\mathrm{abcdA}}$ & $\pm 1.73^{\text {bcdAB }}$ & $\pm 0.25^{\mathrm{abcdAB}}$ & $\pm 0.49^{\mathrm{abcdA}}$ & $\pm 0.58^{\mathrm{bcdA}}$ & $\pm 1.07^{\mathrm{bcdA}}$ & $\pm 0.58^{\mathrm{cdAB}}$ & $\pm 0.10^{\mathrm{bcdB}}$ & $\pm 0.12^{\text {bcdAB }}$ \\
\hline & & & & ----- & $\operatorname{Mean} \pm \mathbf{S E}^{*} ; 2$ & $\left.\mathbf{w k}^{* *} \mathbf{g}\right)$---- & & & \\
\hline \multirow[t]{2}{*}{ Imported } & 38.60 & 34.13 & 32.40 & 34.09 & 30.43 & 30.50 & 32.90 & 31.16 & 27.30 \\
\hline & $\pm 1.20^{\mathrm{bcdA}}$ & $\pm 0.58^{\mathrm{abcA}}$ & $\pm 1.15^{\mathrm{abcdA}}$ & $\pm 3.12^{\mathrm{abcA}}$ & $\pm 2.31^{\mathrm{bcdA}}$ & $\pm 2.44^{\mathrm{bcdA}}$ & $\pm 3.31^{\mathrm{abcdA}}$ & $\pm 0.88^{\mathrm{bcdA}}$ & $\pm 3.60^{\mathrm{dA}}$ \\
\hline \multirow[t]{2}{*}{ Local-1 } & 29.35 & 32.48 & 34.53 & 34.41 & 28.82 & 38.60 & 32.08 & 32.68 & 29.47 \\
\hline & $\pm 0.87^{\mathrm{cdA}}$ & $\pm 1.47^{\mathrm{abcdA}}$ & $\pm 1.96^{\mathrm{abcA}}$ & $\pm 2.06^{\mathrm{abcA}}$ & $\pm 2.46^{\mathrm{cdA}}$ & $\pm 1.20^{\mathrm{Ab}}$ & $\pm 0.28^{\mathrm{bcdA}}$ & $\pm 1.84^{\mathrm{abcdA}}$ & $\pm 0.89^{\mathrm{cdA}}$ \\
\hline \multirow[t]{2}{*}{ Local-2 } & 35.17 & 29.07 & 32.09 & 34.37 & 32.69 & 32.70 & 31.69 & 28.80 & 30.02 \\
\hline & $\pm 1.11^{\mathrm{abcB}}$ & $\pm 3.71^{\mathrm{cdB}}$ & $\pm 1.99^{\mathrm{bcdA}}$ & $\pm 0.80^{\mathrm{abcA}}$ & $\pm 2.32^{\mathrm{abcdA}}$ & $\pm 1.37^{\mathrm{abcdAB}}$ & $\pm 0.40^{\mathrm{bcdA}}$ & $\pm 0.97^{\mathrm{cdB}}$ & $\pm 1.66^{\mathrm{cdAB}}$ \\
\hline \multirow[t]{3}{*}{ Local-3 } & 34.50 & 31.61 & 32.98 & 31.92 & 28.59 & 29.74 & 36.66 & 30.57 & 32.50 \\
\hline & $\pm 1.22^{\mathrm{abcAB}}$ & $\pm 2.41^{\mathrm{bcdA}}$ & $\pm 1.09^{\mathrm{abcdA}}$ & $\pm 1.69^{\mathrm{bcdB}}$ & $\pm 2.25^{\mathrm{cdB}}$ & $\pm 2.46^{\mathrm{cdAB}}$ & $\pm 1.47^{\mathrm{abAB}}$ & $\pm 0.73^{\text {bcdAB }}$ & $\pm 0.64^{\mathrm{abcd} A \mathrm{~B}}$ \\
\hline & & & & 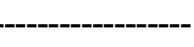 & $\operatorname{Mean} \pm \mathrm{SE}^{*}$ & $w^{* *}$ g) ---. & 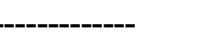 & & \\
\hline \multirow[t]{2}{*}{ Imported } & 46.73 & 40.03 & 44.49 & 51.90 & 45.76 & 43.30 & 52.83 & 44.40 & 41.13 \\
\hline & $\pm 1.82^{\mathrm{abcA}}$ & $\pm 3.11^{\mathrm{cdA}}$ & $\pm 2.94^{\mathrm{bcdA}}$ & $\pm 4.45^{\mathrm{abA}}$ & $\pm 5.28^{\mathrm{abcdA}}$ & $\pm 0.85^{\mathrm{cdA}}$ & $\pm 2.42^{\mathrm{aA}}$ & $\pm 1.30^{\mathrm{bcdA}}$ & $\pm 1.62^{\mathrm{cdA}}$ \\
\hline \multirow[t]{2}{*}{ Local-1 } & 44.00 & 46.33 & 40.70 & 43.06 & 42.00 & 41.16 & 44.13 & 43.16 & 39.92 \\
\hline & $\pm 0.57^{\mathrm{cdA}}$ & $\pm 0.83^{\mathrm{abcdB}}$ & $\pm 0.97^{\mathrm{cdA}}$ & $\pm 1.43^{\mathrm{cdB}}$ & $\pm 1.52^{\mathrm{cdB}}$ & $\pm 3.34^{\mathrm{cdA}}$ & $\pm 2.29^{\mathrm{cdB}}$ & $\pm 0.52^{\mathrm{cdA}}$ & $\pm 2.70^{\mathrm{cdA}}$ \\
\hline \multirow[t]{2}{*}{ Local-2 } & 42.80 & 43.73 & 40.96 & 45.60 & 42.80 & 42.93 & 44.66 & 40.43 & 38.63 \\
\hline & $\pm 2.50^{\mathrm{cdA}}$ & $\pm 0.13^{\mathrm{cdAB}}$ & $\pm 1.22^{\mathrm{cdA}}$ & $\pm 4.32^{\mathrm{abcd} A \mathrm{~B}}$ & $\pm 1.67^{\mathrm{cdAB}}$ & $\pm 2.05^{\mathrm{cdA}}$ & $\pm 0.65^{\mathrm{bcdAB}}$ & $\pm 2.91^{\mathrm{cdA}}$ & $\pm 1.27^{\mathrm{dB}}$ \\
\hline \multirow[t]{3}{*}{ Local-3 } & 44.33 & 42.60 & 42.73 & 46.56 & 41.03 & 41.76 & 45.91 & 42.43 & 41.36 \\
\hline & $\pm 0.92^{\mathrm{bcdA}}$ & $\pm 1.51^{\mathrm{cdAB}}$ & $\pm 1.49^{\mathrm{cdA}}$ & $\pm 4.08^{\mathrm{abcd} A \mathrm{~B}}$ & $\pm 1.18^{\mathrm{cdAB}}$ & $\pm 1.49^{\mathrm{cdA}}$ & $\pm 1.58^{\mathrm{abcd} A \mathrm{~B}}$ & $\pm 0.72^{\mathrm{cdA}}$ & $\pm 1.02^{\mathrm{cdAB}}$ \\
\hline & & & & & ean $\pm \mathrm{SE}^{*}$ & $\left.{ }^{* *} \operatorname{cum}^{\dagger} \mathbf{g}\right)$ & & & \\
\hline \multirow[t]{2}{*}{ Imported } & 109.54 & 100.58 & 94.85 & 103.00 & 92.02 & 88.18 & 97.95 & 98.14 & 89.12 \\
\hline & $\pm 1.09^{\mathrm{Aa}}$ & $\pm 1.68^{\mathrm{abcdA}}$ & $\pm 4.48^{\operatorname{cdefg} A}$ & $\pm 7.14^{\mathrm{abcA}}$ & $\pm 6.46^{\text {cdefghijA }}$ & $\pm 3.81^{\text {defghijA }}$ & $\pm 1.81^{\text {bcdeA }}$ & $\pm 3.31^{\mathrm{bcdeA}}$ & $\pm 4.85^{\text {defghijA }}$ \\
\hline \multirow[t]{2}{*}{ Local-1 } & 96.99 & 91.49 & 95.07 & 92.80 & 97.34 & 93.10 & 94.17 & 89.66 & 89.38 \\
\hline & $\pm 5.44^{\text {bcdefB }}$ & $\pm 8.13^{\text {cdefghijB }}$ & $\pm 1.82^{\text {bcdefg } \mathrm{A}}$ & $\pm 3.71^{\text {bcdefghiB }}$ & $\pm 1.04^{\mathrm{bcdeB}}$ & $\pm 1.09^{\text {bcdefghiB }}$ & $\pm 4.50^{\text {bcdefghB }}$ & $\pm 2.92^{\text {defghijAB }}$ & $\pm 3.19^{\operatorname{defghij} A}$ \\
\hline \multirow[t]{2}{*}{ Local-2 } & 92.60 & 96.73 & 93.92 & 82.88 & 104.76 & 85.74 & 79.76 & 96.83 & 94.44 \\
\hline & $\pm 2.05^{\text {bcdefghiBC }}$ & $\pm 0.78^{\text {bcdefAB }}$ & $\pm 2.94^{\text {bcdefghB }}$ & $\pm 1.78^{\text {ghijkBC }}$ & $\pm 2.61^{\mathrm{abBC}}$ & $\pm 4.59^{\text {efghijkABC }}$ & $\pm 0.72^{\mathrm{jkC}}$ & $\pm 2.87^{\text {bcdefAB }}$ & $\pm 2.10^{\text {bcdefghB }}$ \\
\hline \multirow[t]{2}{*}{ Local-3 } & 87.12 & 84.46 & 83.42 & 81.03 & 81.94 & 76.20 & 90.82 & 86.61 & 83.60 \\
\hline & $\pm 2.60^{\text {efghijkD }}$ & $\pm 1.51^{\text {fghijkBC }}$ & $\pm 2.32^{\text {ghijkBC }}$ & $\pm 4.51^{\mathrm{ijkBC}}$ & $\pm 4.13^{\text {hijkAD }}$ & $\pm 3.40^{\mathrm{kC}}$ & $\pm 3.54^{\text {cdefghij } A B C}$ & $\pm 3.98^{\text {efghijkABC }}$ & $\pm 0.84^{\text {ghijkABC }}$ \\
\hline
\end{tabular}

Different small alphabets on means in a row show significant differences at $\mathrm{p}<0.05$

Different capital alphabets on means in a column show significant differences at $\mathrm{p}<0.05$

${ }^{*} \mathrm{SE}=$ Standard error

${ }^{* *} \mathrm{wk}=$ Week

cum $=$ Cumulative 
Table-4. $1^{\text {st }}, 2^{\text {nd }}, 3$ rd week and cumulative progeny feed intake (g) influenced by 3 parental body weight categories from 4 close-bred flocks of Japanese quails.

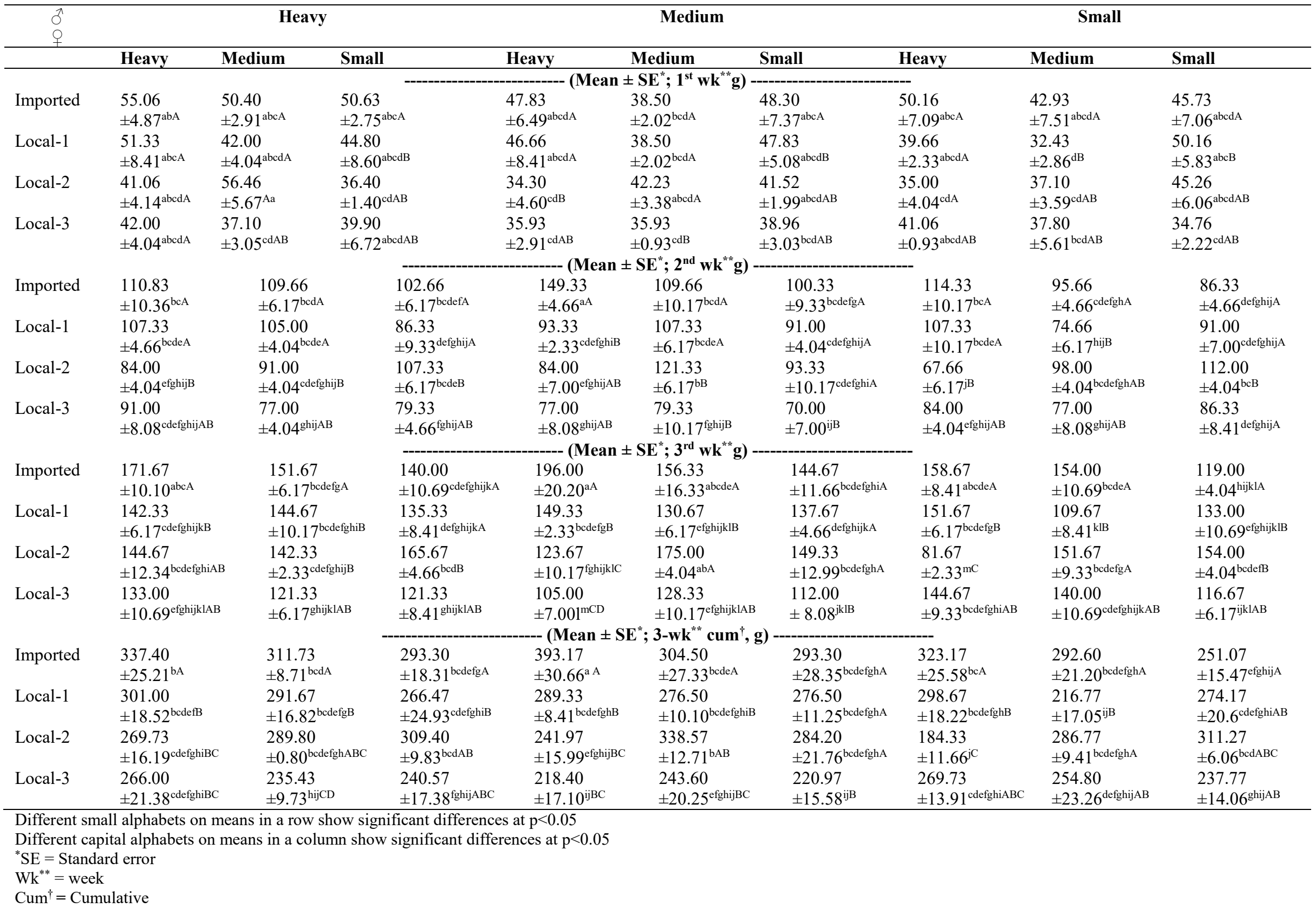


Jatoi et al.,

The J. Anim. Plant Sci. 31(2):2021

Table-5. $1^{\text {st }}, 2^{\text {nd }}, 3^{\text {rd }}$ week and cumulative progeny feed conversion ratio (FCR $)$ influenced by 3 parental body weight categories from 4 close-bred flocks of Japanese quails.

\begin{tabular}{|c|c|c|c|c|c|c|c|c|c|}
\hline \multirow{2}{*}{$\begin{array}{l}\hat{0} \\
0\end{array}$} & \multicolumn{3}{|c|}{ Heavy } & \multicolumn{3}{|c|}{ Medium } & \multicolumn{3}{|c|}{ Small } \\
\hline & Heavy & Medium & Small & Heavy & Medium & Small & Heavy & Medium & Small \\
\hline \multirow{3}{*}{ Imported } & & & & (Mean & , 1st wk $\left.{ }^{* * *}\right)$ & & & & \\
\hline & 2.77 & 2.48 & 2.57 & 2.68 & 1.83 & 2.74 & 2.50 & 2.26 & 2.39 \\
\hline & $\pm 0.12^{\mathrm{abA}}$ & $\pm 0.02^{\mathrm{abA}}$ & $\pm 0.18^{\mathrm{abA}}$ & $\pm 0.51^{\mathrm{abA}}$ & $\pm 0.15^{\mathrm{bA}}$ & $\pm 0.54^{\mathrm{abA}}$ & $\pm 0.39^{\mathrm{abA}}$ & $\pm 0.60^{\mathrm{bA}}$ & $\pm 0.49^{\mathrm{bA}}$ \\
\hline \multirow[t]{2}{*}{ Local-1 } & 2.85 & 2.42 & 2.39 & 2.49 & 2.04 & 2.48 & 2.20 & 1.69 & 2.86 \\
\hline & $\pm 0.64^{\mathrm{abA}}$ & $\pm 0.21^{\mathrm{abA}}$ & $\pm 0.50^{\mathrm{bB}}$ & $\pm 0.43^{\mathrm{abA}}$ & $\pm 0.18^{\mathrm{bA}}$ & $\pm 0.30^{\mathrm{abA}}$ & $\pm 0.07^{\mathrm{bA}}$ & $\pm 0.10^{\mathrm{bA}}$ & $\pm 0.29^{\mathrm{abA}}$ \\
\hline \multirow[t]{2}{*}{ Local-2 } & 2.11 & 3.54 & 2.31 & 1.77 & 2.32 & 2.22 & 2.10 & 2.24 & 2.74 \\
\hline & $\pm 0.27^{\mathrm{bA}}$ & $\pm 0.63^{\mathrm{aB}}$ & $\pm 0.36^{\mathrm{bB}}$ & $\pm 0.20^{\mathrm{bB}}$ & $\pm 0.18^{\mathrm{bA}}$ & $\pm 0.07^{\mathrm{bA}}$ & $\pm 0.28^{\mathrm{bA}}$ & $\pm 0.26^{\mathrm{bA}}$ & $\pm 0.47^{\mathrm{abA}}$ \\
\hline \multirow[t]{3}{*}{ Local-3 } & 2.34 & 2.21 & 2.06 & 2.00 & 2.05 & 2.22 & 2.44 & 2.22 & 2.03 \\
\hline & $\pm 0.19^{\mathrm{bA}}$ & $\pm 0.27^{\mathrm{bAB}}$ & $\pm 0.31^{\mathrm{bB}}$ & $\pm 0.11^{\mathrm{bAB}}$ & $\pm 0.09^{\mathrm{bA}}$ & $\pm 0.12^{\mathrm{bA}}$ & $\pm 0.13^{\mathrm{abA}}$ & $\pm 0.34^{\mathrm{bA}}$ & $\pm 0.11^{\mathrm{bA}}$ \\
\hline & & & & ---- (Mea & , $2 \mathrm{nd} \mathrm{wk}^{* * *}$ & & & & \\
\hline \multirow[t]{2}{*}{ Imported } & 3.56 & 3.21 & 3.18 & 4.46 & 3.69 & 3.38 & 3.62 & 3.07 & 3.24 \\
\hline & $\pm 0.31^{\mathrm{abcdA}}$ & $\pm 0.15^{\text {bcdefgA }}$ & $\pm 0.27^{\text {bcdefg } \mathrm{A}}$ & $\pm 0.50^{\mathrm{bA}}$ & $\pm 0.60^{\mathrm{abcA}}$ & $\pm 0.59^{\text {bcdeA }}$ & $\pm 0.72^{\text {abcdA }}$ & $\pm 0.12^{\text {bcdefghA }}$ & $\pm 0.29^{\text {bcdefg } \mathrm{A}}$ \\
\hline \multirow[t]{2}{*}{ Local-1 } & 3.65 & 3.25 & 2.49 & 2.73 & 3.76 & 2.36 & 3.34 & 2.28 & 3.08 \\
\hline & $\pm 0.14^{\mathrm{abcdA}}$ & $\pm 0.26^{\text {bcdefg } A}$ & $\pm 0.19^{\text {efghB }}$ & $\pm 0.18^{\text {bcdefghB }}$ & $\pm 0.31^{\mathrm{abA}}$ & $\pm 0.16^{\text {efghB }}$ & $\pm 0.32^{\text {bcdefgB }}$ & $\pm 0.17^{\text {bcdefghA }}$ & $\pm 0.14^{\text {bcdefgh } \mathrm{A}}$ \\
\hline \multirow[t]{2}{*}{ Local-2 } & 2.39 & 3.19 & 3.34 & 2.45 & 3.72 & 2.89 & 2.13 & 3.41 & 3.76 \\
\hline & $\pm 0.18^{\text {efghB }}$ & $\pm 0.25^{\text {bcdefgA }}$ & $\pm 0.08^{\text {bcdefAB }}$ & $\pm 0.23^{\text {efgh } A B}$ & $\pm 0.07^{\mathrm{abA}}$ & $\pm 0.43^{\text {bcdefghB }}$ & $\pm 0.20^{\mathrm{hC}}$ & $\pm 0.22^{\text {bcdeA }}$ & $\pm 0.32^{\mathrm{abB}}$ \\
\hline \multirow[t]{3}{*}{ Local-3 } & 2.62 & 2.44 & 2.41 & 2.41 & 2.76 & 2.40 & 2.30 & 2.51 & 2.65 \\
\hline & $\pm \mathrm{d} 0.14^{\mathrm{efghB}}$ & $\pm 0.07^{\text {efghB }}$ & $\pm 0.16^{\text {efghAB }}$ & $\pm 0.27^{\mathrm{efghAB}}$ & $\pm 0.21^{\text {bcdefghB }}$ & $\pm 0.36^{\text {efgh } A B}$ & $\pm 0.16^{\mathrm{ghAC}}$ & $\pm 0.22^{\text {efghA }}$ & $\pm 0.27^{\text {cdefgh } \mathrm{AB}}$ \\
\hline & & & & (Mean & , 3 rd wk $\left.{ }^{* * * *}\right)$ & & & & \\
\hline \multirow[t]{2}{*}{ Imported } & 3.66 & 3.85 & 3.16 & 3.77 & 3.45 & 3.35 & 3.01 & 3.46 & 2.89 \\
\hline & $\pm 0.09^{\text {abcdefA }}$ & $\pm 0.45^{\mathrm{abcdA}}$ & $\pm 0.25^{\text {defgh } A}$ & $\pm 0.16^{\mathrm{abcdeA}}$ & $\pm 0.31^{\text {abcdefg } \mathrm{A}}$ & $\pm 0.32^{\text {abcdefg } A}$ & $\pm 0.17^{\text {efghiA }}$ & $\pm 0.14^{\text {abcdefg } A}$ & $\pm 0.03^{\text {fghiA }}$ \\
\hline \multirow[t]{2}{*}{ Local-1 } & 3.23 & 3.12 & 3.31 & 3.47 & 3.10 & 3.41 & 3.46 & 2.53 & 3.34 \\
\hline & $\pm 0.11^{\text {cdefghA }}$ & $\pm 0.22^{\text {defghB }}$ & $\pm 0.12^{\text {bcdefghAB }}$ & $\pm 0.17^{\text {abcdefg } A B}$ & $\pm 0.03^{\operatorname{defgh} A B}$ & $\pm 0.42^{\text {abcdefg } A}$ & $\pm 0.31^{\text {abcdefg } A B}$ & $\pm 0.17^{\text {hiB }}$ & $\pm 0.25^{\text {abcdefB }}$ \\
\hline \multirow[t]{2}{*}{ Local-2 } & 3.40 & 3.25 & 4.04 & 2.78 & 4.09 & 3.48 & 1.82 & 3.75 & 3.99 \\
\hline & $\pm 0.31^{\text {abcdefg } A}$ & $\pm 0.05^{\text {bcdefghAB }}$ & $\pm 0.01^{\mathrm{abABC}}$ & $\pm 0.42^{\text {ghiBC }}$ & $\pm 0.12^{\mathrm{aA}}$ & $\pm 0.30^{\text {abcdefg } A}$ & $\pm 0.06^{\mathrm{jC}}$ & $\pm 0.09^{\mathrm{abcde} A}$ & $\pm 0.17^{\mathrm{abcB}}$ \\
\hline \multirow[t]{3}{*}{ Local-3 } & 3.00 & 2.85 & 2.84 & 2.26 & 3.14 & 2.70 & 3.14 & 3.29 & 2.82 \\
\hline & $\pm 0.23^{\text {efghiA }}$ & $\pm 0.18^{\mathrm{ghiABC}}$ & $\pm 0.21^{\text {ghiABCD }}$ & $\pm 0.09^{\mathrm{iBC}}$ & $\pm 0.32^{\operatorname{defghAB}}$ & $\pm 0.28^{\text {ghiAB }}$ & $\pm 0.14^{\operatorname{defgh} A B}$ & $\pm 0.20^{\text {bcdefgh } A B}$ & $\pm 0.13^{\text {ghiAB }}$ \\
\hline & & & & (Mean \pm & , 3-wk $\mathbf{k}^{* * *} \operatorname{cum}^{\dagger}$ & - & & & \\
\hline \multirow[t]{2}{*}{ Imported } & 3.07 & 3.10 & 3.08 & 3.81 & 3.31 & 3.32 & 3.30 & 2.97 & 2.81 \\
\hline & $\pm 0.20^{\mathrm{bcdA}}$ & $\pm 0.10^{\mathrm{bcdA}}$ & $\pm 0.06^{\text {bcdA }}$ & $\pm 0.05^{\mathrm{aA}}$ & $\pm 0.20^{\mathrm{bA}}$ & $\pm 0.1^{\mathrm{bA}}$ & $\pm 0.26^{\mathrm{bcA}}$ & $\pm 0.12^{\mathrm{bcdA}}$ & $\pm 0.02^{\text {bcdeA }}$ \\
\hline \multirow[t]{2}{*}{ Local-1 } & 3.10 & 3.20 & 2.80 & 3.13 & 2.83 & 2.97 & 3.16 & 2.41 & 3.06 \\
\hline & $\pm 0.11^{\mathrm{bcdA}}$ & $\pm 0.12^{\mathrm{bcA}}$ & $\pm 0.28^{\mathrm{bcdeB}}$ & $\pm 0.21^{\mathrm{bcdB}}$ & $\pm 0.08^{\mathrm{bcdeB}}$ & $\pm 0.12^{\mathrm{bcdB}}$ & $\pm 0.05^{\mathrm{bcdB}}$ & $\pm 0.11^{\mathrm{efB}}$ & $\pm 0.17^{\mathrm{bcdB}}$ \\
\hline \multirow[t]{2}{*}{ Local-2 } & 2.90 & 2.99 & 3.29 & 2.91 & 3.23 & 3.32 & 2.30 & 2.96 & 3.30 \\
\hline & $\pm 0.11^{\mathrm{bcdA}}$ & $\pm 0.02^{\mathrm{bcdA}}$ & $\pm 0.02^{\mathrm{bcAB}}$ & $\pm 0.17^{\mathrm{bcdB}}$ & $\pm 0.04^{\mathrm{bcAB}}$ & $\pm 0.21^{\mathrm{bA}}$ & $\pm 0.13^{\mathrm{fC}}$ & $\pm 0.01^{\mathrm{bcdA}}$ & $\pm 0.13^{\mathrm{bcAB}}$ \\
\hline \multirow[t]{2}{*}{ Local-3 } & 3.04 & 2.78 & 2.87 & 2.69 & 2.96 & 2.90 & 2.96 & 2.93 & 2.84 \\
\hline & $\pm 0.15^{\mathrm{bcdA}}$ & $\pm 0.07^{\mathrm{cde} A \mathrm{~B}}$ & $\pm 0.12^{\text {bcdeB }}$ & $\pm 0.10^{\operatorname{defBC}}$ & $\pm 0.13^{\mathrm{bcdAB}}$ & $\pm 0.18^{\mathrm{bcdB}}$ & $\pm 0.13^{\text {bcdAB }}$ & $\pm 0.21^{\mathrm{bcdA}}$ & $\pm 0.18^{\text {bcdeAB }}$ \\
\hline
\end{tabular}

Different small alphabets on means in a row show significant differences at $\mathrm{p}<0.05$

Different capital alphabets on means in a column show significant differences at $p<0.05$

${ }^{*} \mathrm{FCR}=$ Feed conversion ratio

${ }^{* *} \mathrm{SE}=$ Standard error

$\mathrm{wk}^{* * *}=\mathrm{WK}=$ Week

cum $^{\dagger}=$ Cumulative 



quails.

\begin{tabular}{|c|c|c|c|c|c|c|c|c|c|}
\hline \multirow{2}{*}{$\begin{array}{l}1 \\
0 \\
0 \\
\end{array}$} & \multicolumn{3}{|c|}{ Heavy } & \multicolumn{3}{|c|}{ Medium } & \multicolumn{3}{|c|}{ Small } \\
\hline & Heavy & Medium & Small & Heavy & Medium & Small & Heavy & Medium & Small \\
\hline Imported & $\begin{array}{l}27.47 \\
\pm 5.88^{\text {abcdefghA }}\end{array}$ & $\begin{array}{l}19.82 \\
\pm 3.10^{\text {cdefghiA }}\end{array}$ & $\begin{array}{l}26.05 \\
\pm 2.33^{\text {abcdefghA }}\end{array}$ & $\begin{array}{l}21.18 \\
\pm 2.76^{\text {abcdefghiA }}\end{array}$ & $\begin{array}{l}12.42 \\
\pm 1.05^{\mathrm{hiA}}\end{array}$ & $\begin{array}{l}28.40 \\
\pm 1.14^{\text {abcdefg } \mathrm{A}}\end{array}$ & $\begin{array}{l}13.76 \\
\pm 2.14^{\mathrm{ghiA}}\end{array}$ & $\begin{array}{l}24.91 \\
\pm 6.71^{\text {abcdefghA }}\end{array}$ & $\begin{array}{l}21.55 \\
\pm 2.92^{\text {abcdefghiA }}\end{array}$ \\
\hline Local-1 & $\begin{array}{l}16.76 \\
\pm 3.69^{\text {efghiA }}\end{array}$ & $\begin{array}{l}18.28 \\
\pm 3.73^{\text {defghiA }}\end{array}$ & $\begin{array}{l}31.73 \\
\pm 6.08^{\text {abcdeA }}\end{array}$ & $\begin{array}{l}22.77 \\
\pm 4.23^{\text {abcdefghA }}\end{array}$ & $\begin{array}{l}19.38 \\
\pm 2.13^{\text {defghiA }}\end{array}$ & $\begin{array}{l}36.40 \\
\pm 2.65^{\mathrm{aA}}\end{array}$ & $\begin{array}{l}24.82 \\
\pm 3.89^{\text {abcdefgh } A B}\end{array}$ & $\begin{array}{l}19.85 \\
\pm 5.14^{\text {cdefghiA }}\end{array}$ & $\begin{array}{l}31.53 \\
\pm 5.83^{\text {abcdefA }}\end{array}$ \\
\hline Local-2 & $\begin{array}{l}35.14 \\
\pm 9.51^{\mathrm{abcdB}}\end{array}$ & $\begin{array}{l}33.35 \\
\pm 3.69^{\mathrm{abcdA}}\end{array}$ & $\begin{array}{l}13.90 \\
\pm 1.83^{\mathrm{ghiB}}\end{array}$ & $\begin{array}{l}6.96 \\
\pm 2.43^{\mathrm{iB}}\end{array}$ & $\begin{array}{l}21.71 \\
\pm 2.67^{\text {abcdefghiA }}\end{array}$ & $\begin{array}{l}21.58 \\
\pm 2.09^{\text {abcdefghiA }}\end{array}$ & $\begin{array}{l}12.03 \\
\pm 3.12^{\mathrm{iA}}\end{array}$ & $\begin{array}{l}23.10 \\
\pm 0.89^{\text {abcdefgh } A}\end{array}$ & $\begin{array}{l}16.10 \\
\pm 7.64^{\text {fghiA }}\end{array}$ \\
\hline Local-3 & $\begin{array}{l}31.02 \\
\pm 10.13^{\text {abcdefA }}\end{array}$ & $\begin{array}{l}23.29 \\
\pm 4.12^{\text {abcdefghA }}\end{array}$ & $\begin{array}{l}29.91 \\
\pm 4.70^{\text {abcdeAC }}\end{array}$ & $\begin{array}{l}20.53 \\
\pm 2.72^{\text {bcdefghiA }}\end{array}$ & $\begin{array}{l}25.69 \\
\pm 4.22^{\text {abcdefghA }}\end{array}$ & $\begin{array}{l}16.10 \\
\pm 7.64^{\mathrm{aA}}\end{array}$ & $\begin{array}{l}27.16 \\
\pm 2.09^{\text {abcdefgh } A B}\end{array}$ & $\begin{array}{l}35.69 \\
\pm 6.21^{\mathrm{abA}}\end{array}$ & $\begin{array}{l}23.83 \\
\pm 3.44^{\text {abcdefghA }}\end{array}$ \\
\hline & & & & ------ (Mean & $\mathrm{SE}^{*} ; 2^{\text {nd }} \mathrm{wk}^{* *}$ & | -------------- & ----- & & \\
\hline Imported & $\begin{array}{l}8.34 \\
\pm 0.65^{\mathrm{abcdeA}}\end{array}$ & $\begin{array}{l}6.74 \\
\pm 3.31^{\text {abcdeA }}\end{array}$ & $\begin{array}{l}6.62 \\
\pm 2.05^{\text {abcdeA }}\end{array}$ & $\begin{array}{l}6.87 \\
\pm 0.29^{\mathrm{abcdeA}}\end{array}$ & $\begin{array}{l}6.27 \\
\pm 1.71^{\mathrm{abcdeA}}\end{array}$ & $\begin{array}{l}3.74 \\
\pm 3.54^{\mathrm{bcdeA}}\end{array}$ & $\begin{array}{l}5.36 \\
\pm 1.09^{\mathrm{abcdeA}}\end{array}$ & $\begin{array}{l}3.97 \\
\pm 3.33^{\mathrm{bcdeA}}\end{array}$ & $\begin{array}{l}12.57 \\
\pm 2.88^{\mathrm{aA}}\end{array}$ \\
\hline Local-1 & $\begin{array}{l}5.70 \\
\pm 3.18^{\text {abcdeA }}\end{array}$ & $\begin{array}{l}4.29 \\
\pm 1.34^{\text {bcdeA }}\end{array}$ & $\begin{array}{l}1.78 \\
\pm 1.38^{\mathrm{cdeB}}\end{array}$ & $\begin{array}{l}10.67 \\
\pm 2.54^{\mathrm{abB}}\end{array}$ & $\begin{array}{l}0.44 \\
\pm 0.01^{\mathrm{eB}}\end{array}$ & $\begin{array}{l}2.98 \\
\pm 2.76^{\text {bcdeA }}\end{array}$ & $\begin{array}{l}0.83 \\
\pm 0.61^{\mathrm{deB}}\end{array}$ & $\begin{array}{l}8.47 \\
\pm 1.93^{\mathrm{abcdeA}}\end{array}$ & $\begin{array}{l}1.11 \\
\pm 0.37^{\mathrm{deB}}\end{array}$ \\
\hline Local-2 & $\begin{array}{l}10.39 \\
\pm 1.52^{\mathrm{abB}}\end{array}$ & $\begin{array}{l}1.68 \\
\pm 0.88^{\mathrm{cdeB}}\end{array}$ & $\begin{array}{l}3.00 \\
\pm 1.10^{\mathrm{bcdeA}}\end{array}$ & $\begin{array}{l}5.87 \\
\pm 2.48^{\text {abcdeA }}\end{array}$ & $\begin{array}{l}4.84 \\
\pm 2.51^{\text {abcdeA }}\end{array}$ & $\begin{array}{l}1.53 \\
\pm 0.42^{\mathrm{cdeB}}\end{array}$ & $\begin{array}{l}3.00 \\
\pm 0.27^{\text {bcdeA }}\end{array}$ & $\begin{array}{l}2.95 \\
\pm 1.06^{\text {bcdeB }}\end{array}$ & $\begin{array}{l}1.45 \\
\pm 0.80^{\mathrm{deB}}\end{array}$ \\
\hline Local-3 & $\begin{array}{l}9.63 \\
\pm 2.07^{\mathrm{abcAB}}\end{array}$ & $\begin{array}{l}3.71 \\
\pm 3.60^{\text {bcdeAB }}\end{array}$ & $\begin{array}{l}1.32 \\
\pm 1.15^{\mathrm{deAB}}\end{array}$ & $\begin{array}{l}7.07 \\
\pm 3.36^{\mathrm{abcdeA}}\end{array}$ & $\begin{array}{l}3.17 \\
\pm 1.36^{\text {bcde } A B}\end{array}$ & $\begin{array}{l}8.82 \\
\pm 6.49^{\mathrm{abcd} A B}\end{array}$ & $\begin{array}{l}2.68 \\
\pm 0.56^{\mathrm{bcdeA}}\end{array}$ & $\begin{array}{l}4.03 \\
\pm 0.66^{\text {bcdeB }}\end{array}$ & $\begin{array}{l}5.34 \\
\pm 4.06^{\text {abcdeAB }}\end{array}$ \\
\hline & & & & (Mean & $\mathrm{SE}^{*} ; 3^{\text {rd }} \mathbf{w k}^{* *}$ & & --- & & \\
\hline Imported & $\begin{array}{l}2.11 \\
\pm 1.08^{\mathrm{bA}}\end{array}$ & $\begin{array}{l}2.04 \\
\pm 0.72^{\mathrm{aA}}\end{array}$ & $\begin{array}{l}0.86 \\
\pm 0.48^{\mathrm{aA}}\end{array}$ & $\begin{array}{l}0.86 \\
\pm 0.43^{\mathrm{bA}}\end{array}$ & $\begin{array}{l}3.45 \\
\pm 0.93^{\mathrm{bA}}\end{array}$ & $\begin{array}{l}6.06 \\
\pm 0.87^{\mathrm{bA}}\end{array}$ & $\begin{array}{l}1.00 \\
\pm 0.56^{\mathrm{aA}}\end{array}$ & $\begin{array}{l}3.36 \\
\pm 1.36^{\mathrm{bA}}\end{array}$ & $\begin{array}{l}4.98 \\
\pm 1.44^{\mathrm{bA}}\end{array}$ \\
\hline Local-1 & $\begin{array}{l}4.02 \\
\pm 1.18^{\mathrm{bA}}\end{array}$ & $\begin{array}{l}2.77 \\
\pm 2.00^{\mathrm{Bb}}\end{array}$ & $\begin{array}{l}2.80 \\
\pm 0.66^{\mathrm{bB}}\end{array}$ & $\begin{array}{l}1.81 \\
\pm 0.40^{\mathrm{bA}}\end{array}$ & $\begin{array}{l}2.67 \\
\pm 1.33^{\mathrm{bA}}\end{array}$ & $\begin{array}{l}2.83 \\
\pm 0.27^{\mathrm{bA}}\end{array}$ & $\begin{array}{l}1.62 \\
\pm 1.14^{\mathrm{bB}}\end{array}$ & $\begin{array}{l}6.36 \\
\pm 0.95^{\mathrm{bA}}\end{array}$ & $\begin{array}{l}1.28 \\
\pm 0.74^{\mathrm{bA}}\end{array}$ \\
\hline Local-2 & $\begin{array}{l}1.38 \\
\pm 1.38^{\mathrm{bA}}\end{array}$ & $\begin{array}{l}1.06 \\
\pm 0.60^{\mathrm{bB}}\end{array}$ & $\begin{array}{l}2.88 \\
\pm 1.19^{\mathrm{bB}}\end{array}$ & $\begin{array}{l}3.69 \\
\pm 2.07^{\mathrm{bA}}\end{array}$ & $\begin{array}{l}2.54 \\
\pm 1.62^{\mathrm{bA}}\end{array}$ & $\begin{array}{l}0.92 \\
\pm 0.92^{\mathrm{bA}}\end{array}$ & $\begin{array}{l}0.77 \\
\pm 0.77^{\mathrm{bB}}\end{array}$ & $\begin{array}{l}1.45 \\
\pm 1.45^{\mathrm{bA}}\end{array}$ & $\begin{array}{l}1.88 \\
\pm 0.77^{\mathrm{bA}}\end{array}$ \\
\hline Local-3 & $\begin{array}{l}3.62 \\
\pm 2.75^{\mathrm{bA}}\end{array}$ & $\begin{array}{l}5.13 \\
\pm 2.85^{\mathrm{bB}}\end{array}$ & $\begin{array}{l}2.40 \\
\pm 1.29^{\mathrm{bB}}\end{array}$ & $\begin{array}{l}5.33 \\
\pm 3.21^{\mathrm{bA}}\end{array}$ & $\begin{array}{l}5.42 \\
\pm 3.17^{\mathrm{bA}}\end{array}$ & $\begin{array}{l}12.34 \\
\pm 4.25^{\mathrm{aB}}\end{array}$ & $\begin{array}{l}2.11 \\
\pm 1.30^{\mathrm{bB}}\end{array}$ & $\begin{array}{l}3.87 \\
\pm 0.56^{\mathrm{bA}}\end{array}$ & $\begin{array}{l}4.48 \\
\pm 3.19^{\mathrm{bA}}\end{array}$ \\
\hline Imported & $\begin{array}{l}37.93 \\
\pm 6.43^{\text {bcdefgA }}\end{array}$ & $\begin{array}{l}28.60 \\
\pm 6.51^{\text {bcdefghijA }}\end{array}$ & $\begin{array}{l}33.54 \\
\pm 1.78^{\text {bcdefghijA }}\end{array}$ & $\begin{array}{l}28.92 \\
\pm 2.92^{\text {bcdefghijA }}\end{array}$ & $\begin{array}{l}\mathbf{E}^{*} ; \mathbf{3}-\mathbf{w k} \mathbf{k}^{* *} \text { cum } \\
22.14 \\
\pm 0.50^{\text {fghijA }}\end{array}$ & $\begin{array}{l}\text { \%) ---------- } \\
38.21 \\
\pm 3.77^{\text {bdefget }}\end{array}$ & $\begin{array}{l}20.13 \\
\pm 2.80^{\text {ghijA }}\end{array}$ & $\begin{array}{l}32.26 \\
\pm 5.06^{\text {bcdefghiA }}\end{array}$ & $\begin{array}{l}39.11 \\
\pm 1.91^{\text {bcdefA }}\end{array}$ \\
\hline Local-1 & $\begin{array}{l}26.49 \\
\pm 7.05^{\text {defghijA }}\end{array}$ & $\begin{array}{l}25.35 \\
\pm 0.38^{\text {defghijB }}\end{array}$ & $\begin{array}{l}36.32 \\
\pm 8.06^{\text {bcdefgA }}\end{array}$ & $\begin{array}{l}35.25 \\
\pm 3.66^{\text {bcdefghB }}\end{array}$ & $\begin{array}{l}22.49 \\
\pm 0.83^{\text {fghijA }}\end{array}$ & $\begin{array}{l}42.22 \\
\pm 2.55^{\text {abcdeA }}\end{array}$ & $\begin{array}{l}27.28 \\
\pm 2.68^{\text {cdefghijB }}\end{array}$ & $\begin{array}{l}34.70 \\
\pm 8.00^{\text {bcdefghiA }}\end{array}$ & $\begin{array}{l}33.93 \\
\pm 6.80^{\text {bcdefghijA }}\end{array}$ \\
\hline Local-2 & $\begin{array}{l}46.92 \\
\pm 7.11^{\mathrm{abB}}\end{array}$ & $\begin{array}{l}36.10 \\
\pm 2.75^{\text {bcdefgh }}\end{array}$ & $\begin{array}{l}19.80 \\
\pm 2.56^{\text {ghijB }}\end{array}$ & $\begin{array}{l}16.54 \\
\pm 5.25^{\mathrm{ijC}}\end{array}$ & $\begin{array}{l}29.10 \\
\pm 2.30^{\text {bcdefghijB }}\end{array}$ & $\begin{array}{l}24.05 \\
\pm 2.82^{\text {efghijB }}\end{array}$ & $\begin{array}{l}15.81 \\
\pm 3.17^{\mathrm{jC}}\end{array}$ & $\begin{array}{l}27.51 \\
\pm 2.72^{\text {cdefghijB }}\end{array}$ & $\begin{array}{l}19.44 \\
\pm 8.30^{\text {hijB }}\end{array}$ \\
\hline Local-3 & $\begin{array}{l}44.29 \\
\pm 7.06^{\mathrm{abcAB}}\end{array}$ & $\begin{array}{l}32.15 \\
\pm 8.86^{\text {bcdefghijAB }}\end{array}$ & $\begin{array}{l}33.64 \\
\pm 2.57^{\text {bcdefghijAB }}\end{array}$ & $\begin{array}{l}32.94 \\
\pm 7.87^{\text {bcdefghijABC }}\end{array}$ & $\begin{array}{l}34.30 \\
\pm 2.70^{\text {bcdefghiB }}\end{array}$ & $\begin{array}{l}57.65 \\
\pm 10.83^{\mathrm{aAB}}\end{array}$ & $\begin{array}{l}31.96 \\
\pm 1.20^{\text {bcdefghijBC }}\end{array}$ & $\begin{array}{l}43.60 \\
\pm 7.22^{\text {abcdAB }}\end{array}$ & $\begin{array}{l}33.66 \\
\pm 5.12^{\text {bcdefghij } A B}\end{array}$ \\
\hline
\end{tabular}

Different small alphabets on means in a row show significant differences at $p<0.05$

Different capital alphabets on means in a column show significant differences at $\mathrm{p}<0.05$

${ }^{*} \mathrm{SE}=$ Standard error

${ }^{* *} \mathrm{WK}=$ Week

${ }^{\dagger} \mathrm{CM}=$ Cumulative 
Conclusion: The day-old and subsequent body weight, weight gain and feed intake were higher in imported than local flocks. The lower feed intake, better feed conversion ratio (FCR) and higher mortality rate were recorded in local-3 as compared to other flocks. The progeny secured from heavy male parent had higher hatch weight, body weight, weight gain and feed intake than those hatched from medium and small male parents, showing more pronounced effect of male parent on progeny growth and on almost all the other parameters.

Acknowledgements: The authors thankfully acknowledge provision of excellent research facilities during course of this study by Avian Research and Training Centre, Department of Poultry Production, Faculty of Animal Production and Technology, University of Veterinary and Animal Sciences, Lahore, Pakistan.

\section{REFERENCES}

Akram M., Z.U. Rehman, A. Mahmood, K. Javed, A.W. Sahota and M.H. Jaspal (2008). Comparative productive performance of Japanese quail from different local and imported flocks. Proceedings of XXIII World Poultry Congress, 30th June4th July, 2008 held at Brisbane, Australia, p. 355.

Alkan, S., K. Karabag, A. Galic, T. Karsli and M. S. Balcioglu (2010). Determination of body weight and some carcass traits in japanese quails (Coturnix coturnix japonica) of different lines. Kafkas. Univ. Vet. Fak. Derg. 16(2): 277-280.

Among, T. K., P. K. Sharma, N. N. Bora and K. K. Baruah (1984). Effect of egg weight and pre incubation storage period on fertility and hatchability of WLH Eggs. Ind. J. Poult. Sci., 19: 108-111.

Daikwo, S.I,. O.M. Momoh, N.I. Dim (2013). Heritability estimates of genetic and phenotypic correlations among some selected carcass traits of Japanese quail (Coturnix coturnix japonica) raised in a sub-humid climate. J. Biol. Agric. \& Healthcare., 13: 60-65.

Decuypere, E. and V. Bruggeman (2007). The endocrine interfaces of environmental and egg factors affecting chick quality. Poult. Sci., 86: 10371042.

Dhaliwal, S. K., M. L. Chaudhary, G. S. Brahand and J. S. Sandhu (2004). Growth and carcass characteristics of selected and control lines of Japanese quails (Coturnix coturnix japonica). Ind. J. Poult. Sci., 39(2): 112-119.

Dogan N., Tulin A and E. Karaman (2010). Genetic parameters of growth curve parameters and weekly body weights in Japanese quails
(Coturnix coturnix japonica). J. Anim. Vet. Adv., 9(3): 501-507.

Dudusola, I.O. (2013). The effect of parental age and egg weight on fertility, hatchability and day-old chick weight of Japanese quail (Cortunix cortunix japonica). Standard Research J. Agricultural Sciences., (2):13-16. http://www.standresjournals.org/journals/SRJAS

Duncan, D. B. (1955). Multiple range and multiple $F$ tests. Biometrics. 11: 1-42.

El-Fiky, F. A., M. A. Aboul-Hassan, S. S. Batta and G. E. Y. Attalah (2000). Comparative study of egg production traits in two strains of Japanese quail. Fayoum J. Agri. Res. \& Dev., 14: 198-205.

Fadhil MA, Hassan KH (2018). Divergent Genetic Selection for Body Weight at Four Weeks Age in Japanese Quail. J. Global Pharma. Technology. 10(11):612-616.

Farooq, M. (1989). The influence of egg weight on hatchability, chick weight and its subsequent performance up to six weeks of age in broilers. M.Sc. (Hons.) Thesis. College of Veterinary Sciences, Lahore, University of Agriculture Faisalabad, Pakistan.

Goodhope, R. G. (1991). First week broiler mortalityInfluence on production. Second Western Meeting of Poultry Clinicians and Pathologists. http://www.westvet.com/1st_week_mortality.ht m Accessed Nov. 7, 2007.

Hartmann, C., K. Johansson, E. Strandberg, L. Rydhmer (2003). Genetic correlations between the maternal genetic effect on chick weight and the direct genetic effects on egg composition traits in a White Leghorn Line. Poult. Sci., 82: 1-8.

Hassan KH (2011). Poultry Breeding. Univ. of Diyala Press. Iraq.

Hussain, J., M. Akram, A. W. Sahota, K. Javed, H.A. Ahmad, S. Mehmood, S. Ahmad, R. Sulaman, I. Rabbani and A. S. Jatoi (2013). Selection for higher three week body weight in Japanese quail: 1. Effect on growth performance. The J. Anim. Plant Sci., 23(6):1496-1500.

Hussen, S.H., A.M. Abdulrahman Al-Khdri and A.M. Hassan (2016). Response to Selection for Body Weight in Japanese Quail (Coturnix coturnix japonica). Iranian J. Appl. Anim. Sci., 6(2):459453.

Iqbal, M., (2011). Growth performance and carcass characteristics in four different varieties of native Aseel. M. Phil. Thesis. Department of Poultry Production, Faculty of Animal Production and Technology, University of Veterinary and Animal Sciences, Lahore, Pakistan.

Jatoi, A. S., A. W. Sahota, M. Akram, K. Javed, M. H. Jaspal, S. Mehmood, J. Hussain, H. M. Ishaq 
and E. Bughio (2015a). Egg quality characteristics as influenced by different body sizes in four close-bred flocks of Japanese quails (Coturnix coturnix japonica). The J. Anim. Plant Sci., 25(4): 921-926.

Jatoi A.S., A.W. Sahota, M. Akram, K. Javed, M.H. Jaspal, J. Hussain, S. Mehmood, Y. Abbas, A.H. Mirani and H.M. Ishaq (2015b). The effect of different body weight categories on plasma macromineral levels in four close-bred flocks of adult Japanese quails (Coturnix coturnix japonica). Turk. J. Vet. Anim. Sci., 39: 343-349.

Joseph, S. N., E. T. Moran Jr. (2005). Effect of flock age and post emergent holding in the hatcher on broiler live performance and further-processing yield. J. Appl. Poult. Res., 14: 512-520.

Khaldari, M. A., H. Pakdel, Mehrabani Yegane, A. Nejati Javaremi and P. Berg (2010). Response to selection and genetic parameters of body and carcass weights in Japanese quail selected for 4week body weight. Poult. Sci., 89:1834-1841.

Krishna, D. and M. Sahitya Rani (2017). Selective Breeding of Japanese Quails for Improvement of Performance. Int. J. Curr. Microbiol. App. Sci. 6(4):2500-2506. doi: https://doi.org/10.20546/ijcmas.2017.604.291

Kumari, P. B., B. R. Gupta, M. G. Prakash and A. R. Reddy (2009). Genetic study on body weights of Japanese quails. Ind. J. of Poult. Sci., 44(3). (Abst).

Lotfi, E., S. Zerehdaran and M. Ahani Azari (2012). Direct and maternal genetic effects of body weight traits in Japanese quail (Coturnix coturnix japonica). Arch. Geflügelk., 76(3): 150-154.

Maiorano, G,. A. Sobolewska, D. Cianciullo, K. Walasik, G. Elminowska-Wenda, A. Sławińska (2012). Influence of in ovo prebiotic and synbiotics administration on meat quality of broiler chickens. Poult. Sci., 91: 2963-2969.

Minvielle, F. (2004). The future of Japanese quail for research and production. W. Poult. Sci. J., 60: 500-507.

Munisi, W.G., S.H. Mbaga and A.M Katule (2015). Evaluation of growth characteristics of parental, $\mathrm{F}_{1}, \mathrm{~F}_{2}$ and backcross chickens from broiler and Black Australorp stocks in Tanzania. Livestock Research for Rural Development 27 (12).

Narinc, D,. T. Aksoy, E. Karaman (2010). Genetic parameters of growth curve parameters and weekly body weights in Japanese quails (Coturnix coturnix japonica). J. Anim. Vet. Adv. 9(3): 501-507. https://doi.org/10.3923/ javaa.2010.501.507
North, M.O., D.D. Bell (1991). Commercial Chicken Production Manual. Published by Avi Book Co. 4th ed. New York. USA.

NRC. (1994). Nutrients requirements of poultry. 9th revised ed., National Academy Press. Washington, D.C., USA.

Okenyi, N., H. M. Ndofor-Foleng, , C. C. Ogbu and C. I. Agu (2013). Genetic parameters and consequences of selection for short-term egg production traits in Japanese quail in a tropical environment. Afr. J. Biotechnol., 12(12): 1357 1362.

Onyewuchi, U.U., I.R. Offor, and C.F. Okoli (2013). Profitability of quail bird and egg production in IMO state. Nig. J. Agric. Food Environ., 9(1): 40-44.

Renden, J. A. and G. R. McDaniel (1984). Egg characteristics and production efficiency of Dwarf $(d w)$ White Leghorn hens divergently selected for body weight. Poult. Sci., 63(2): 214-221.

Rondelli, S., O. Martinez and P. T. Garcia (2003). Sex effect on productive parameters, carcass and body fat composition of two commercial broilers lines. Rev. Bras. Cienc. Avic. 5(3): 169-173.

Saatci, M., H. Omed and I. Ap Dewi (2006). Genetic parameters from univariate and bivariate analysis of egg and weight traits in Japanese quail. Poult. Sci., 85: 185-190.

Saatci, M., I. Ap Dewi and A. R. Aksoy (2003). Application of REML procedure to estimate the genetic parameters of weekly live weights in one-to-one sire and dam pedigree recorded Japanese quail. J. Anim. Breed. Genet. 120: 23 28

Sahota, A. W., B. M. Bhatti and L. A. Akhtar (2003). Comparative productive performance of Desi parent chicken and their first progeny maintained on deep litter system. Pakistan Vet. J., 23(1): 7-10.

Saidu, S., G. Afanasyev, L. Popova, A. Komarchev and U. Ibrahim (2014) Dynamic of Reproductive Qualities of Japanese Quails. International Conference on Earth, Environment and Life Sciences, (EELS-2014) December 23-24, Dubai (UAE).

SAS. (2002-03). SAS/STATE User's guide: Statistics. Version 9.1., SAS Institute Inc, Cary, North Carolina, USA.

Shokoohmand, M., N. E. J. Kashan and M. A. E. Maybody (2007). Estimation of heritability and genetic correlations of body weight in different age for three strains of Japanese quail. Int. J. of Agri. \& Biology. 9(6): 945-947.

Tona, K., V. Bruggeman, O. Onagbesana, F. Bamelis, M. Gbeassor, K. Mertens and E. Decuypere (2005). 
Day-old chick quality: Relationship to hatching egg quality, adequate incubation practice and prediction of broiler performance. Avian Poult. Biol. Rev., 16: 109-119.

Tunsaringkarn, T., Tungjaroenchai, W. and Siriwong, W. (2013) Nutrient benefits of quail (Coturnix coturnix japonica) eggs. Int. J. Sci. Res. Pub., $3(5): 2$.

Udeh, I. and S.I. Omeje (2011). Growth and Short Term Egg Production of Two Exotic (Layer Type) and the Local Chickens Compared with Their F1 Inbred Progenies. Inter. J. Poult. Sci., 10(3): 221-224.

Umar A., S. Khan., Rafiullah., N. Chand., Z. A. Shah., A. Akhtar and A. J. Tanweer. 2013. Effect of male to female ratio and vitamin-e selenium on fertility, hatchability and hatched chick weight of quail breeders. Sarhad. J. Agric. 29(3): 441447.

Varkoohi, S., M. Moradi Shahr Babak, A. Pakdel, A. Nejati Javaremi, M. Zaghari, A. Kause (2010). Response to selection for feed conversion ratio in Japanese quails. Poult. Sci., 89: 1590-1598.
Wang, B., D. Yeye, S. Qiqing, L. Xue, X. Chundi, G. Zhaoyu and C. Xingyong (2019). Comparative study of growth performance and meat quality of three-line crossbred commercial group from Shanzhongxian and W-line chicken. Italian J. Anim. Sci., 18(1):63-69. https://doi.org/10.1080/1828051X.2018.148279 7

Wilson, H. R. (1991). Effect of egg size on hatchability, chick size and post-hatching growth. p. 279-283. In:"Avian Incubation". Tullett SG. ed. Butterworth-Heinemann Ltd., Surrey, UK.

Wilson, H. R. (1991a). Interrelationship of egg size, chick size, post-hatching growth and hatchability. World's Poult. Sci. J., 47: 5-20.

Yakubu, A., J. A. Ayoade and Y. M. Dahiru (2010). Effects of genotype and population density on growth performance, carcass characteristics, and cost-benefits of broiler chickens in north central Nigeria. Trop. Anim. Health Prod. 42:719-727.

Yassin, H., A. G. J. Velthuis, M. Boerjan and J. V. Riel (2009). Field study on broilers' first-week mortality. Poult. Sci., 88: 798-804. 